\title{
ACAROLOGY
}

\section{Influência de Fragmentos de Cerrado na Distribuição de Ácaros em Seringal}

\author{
Peterson R. Demite ${ }^{1}$ e Reinaldo J.F. Feres ${ }^{2}$ \\ ${ }^{1}$ Programa de Pós-graduação em Biologia Animal, UNESP - São José do Rio Preto, SP, Bolsista \\ Plantações E.Michelin Ltda., peterson_demite@yahoo.com.br \\ ${ }^{2}$ Depto. Zoologia e Botânica, Univ. Estadual Paulista - UNESP, 15054-000, São José do Rio Preto, SP \\ reinaldo@ibilce.unesp.br
}

Neotropical Entomology 37(2):196-204 (2008)

Influence of Cerrado Fragments in the Distribution of Mites in Rubber Tree Crop

\begin{abstract}
The aim of this study was to verify whether fragments of cerrado influence the composition of the mite fauna on rubber trees. Five transects distant $50 \mathrm{~m}$, being the first in the edge near the native areas and the last $200 \mathrm{~m}$ inside the crop, were established in each rubber tree crop in southern State of Mato Grosso. In each transect five plants were chosen, and seven leaves were collected from each plant. During one year, 25 quantitative samplings were conducted in two rubber tree crops. The lowest number of phytophagous mites occurred in the transect closer to the native vegetation, and the highest number, in the most distant from the native vegetation. The largest diversity was also observed in the transect closer to the neighboring vegetation. Ten species of predatory mites were also registered in neighboring native areas. These data suggest the movement of predatory mites from the native areas to the monoculture. These natural areas can possibly supply alternative food and habitat for natural enemies of phytophagous mites in the period of food scarceness in the rubber tree crop. The presence of native areas close to culture areas should be taken into account in the elaboration of programs of ecological management of pests.
\end{abstract}

KEY WORDS: Acari, agroecosystem, ecological pest management, Hevea brasiliensis, native area

RESUMO - Este estudo teve como objetivo verificar se fragmentos de cerrado influenciam na composição da acarofauna de seringais. Foram estabelecidos cinco transectos, distantes $50 \mathrm{~m}$ entre si, em duas áreas de cultivo no sul do Mato Grosso, sendo o primeiro na borda com o limite das áreas nativas e o último a $200 \mathrm{~m}$ no interior do seringal. Em cada transecto foram marcadas cinco plantas, sendo coletadas sete folhas de cada planta. Durante um ano foram realizadas 25 coletas quantitativas em dois cultivos de seringueiras. O menor número de fitófagos foi registrado no transecto próximo da vegetação nativa e o maior no mais distante. A maior diversidade também foi observada no transecto mais próximo da vegetação nativa. Dez espécies de ácaros predadores também foram registradas nas áreas nativas vizinhas. Os dados sugerem o deslocamento dos ácaros predadores das áreas naturais para o monocultivo. Essas áreas naturais podem fornecer alimento alternativo e hábitat para inimigos naturais de fitófagos no período de escassez de alimento no seringal. A presença de áreas nativas próximo a áreas de cultivo deve ser considerada na elaboração de programas de manejo ecológico de pragas.

PALAVRAS-CHAVE: Acari, agroecossistema, manejo ecológico de pragas, Hevea brasiliensis, área nativa

Fragmentos de mata nativa podem afetar a distribuição de ácaros em cultivos. Segundo Altieri (1994), as bordas são importantes para a propagação e proteção de ampla gama de agentes naturais de controle biológico de pragas agrícolas. Esses ambientes podem servir como corredores biológicos que permitem o movimento e distribuição de artrópodes benéficos. Estudos documentam a maior abundância de inimigos naturais e controle biológico mais efetivo em culturas próximas de vegetação nativa (Altieri 1999). Essas áreas fornecem hábitat e alimento para inimigos naturais, como pólen e néctar, mantendo essas espécies com recursos sazonais durante os períodos desfavoráveis à sua manutenção nas culturas (Landis et al. 2000).

Em estudo realizado em um seringal (Hevea brasiliensis Muell. Arg.) no município de São José do Rio Preto, SP, a menor incidência do fitófago Calacarus heveae Feres (Actinedida: Eriophyidae) e a maior de ácaros predadores, foi registrada na borda próxima da vegetação nativa, o que sugere ser importante a presença de vegetação natural vizinha às áreas de cultivo, sendo este um fator a ser considerado na 
elaboração de programas de manejo ecológico de pragas (Demite \& Feres 2005).

Demite \& Feres (2007) analisaram a ocorrência sazonal da acarofauna presente em duas áreas de cultivo de seringueira no sul do Mato Grosso. Este trabalho teve como objetivo verificar a influência de fragmentos de cerrado na distribuição e ocorrência da acarofauna nessas mesmas áreas.

\section{Material e Métodos}

O estudo foi desenvolvido em duas áreas de cultivo de seringueira do clone PB 235, vizinhas de dois fragmentos de cerrado (sensu Ribeiro \& Walter 1998): Cerradão (17 $23^{\circ}$ 'S, $\left.54^{\circ} 42^{\prime} \mathrm{W}\right)$ e Mata Ripária ( $\left.17^{\circ} 22^{\prime} \mathrm{S}, 54^{\circ} 41^{\prime} \mathrm{W}\right)$, distantes $1100 \mathrm{~m}$ uma da outra, no município de Itiquira, sul do Mato Grosso.

Foram estabelecidos cinco transectos, distantes $50 \mathrm{~m}$ entre si, desde a borda no limite com as áreas nativas, até 200 $\mathrm{m}$ no interior do seringal. Em cada transecto foram marcadas cinco plantas distantes cerca de $25 \mathrm{~m}$ entre si, totalizando 25 plantas por área de estudo. Em cada uma das 25 coletas, com intervalo médio de 14 dias entre elas, realizadas de março de 2004 a março de 2005 , foram coletadas sete folhas de cada planta ao redor da copa, até a altura de sete a oito metros. As áreas estudadas não receberam nenhum tipo de tratamento químico ou biológico durante o período de desenvolvimento do estudo.

Para o transporte aéreo até São José do Rio Preto, SP, as folhas foram acondicionadas em sacos de papel, no interior de sacos de polietileno, colocados em caixas isotérmicas de poliestireno refrigeradas com gel a base de celulose vegetal $\left(G e l o-X^{\circledR}\right)$ em seu interior. No laboratório, o material foi armazenado sob refrigeração a temperatura de $10^{\circ} \mathrm{C}$, por um período máximo de sete dias. Foi analisado um folíolo de cada folha (totalizando 175 folíolos/área) sendo os ácaros montados em lâminas de microscopia, sob microscópio estereoscópico, utilizando-se o meio de Hoyer (Flechtmann 1975). Os ácaros oribatídeos foram triados e armazenados em álcool etílico a 70\%. Nos períodos de grandes índices populacionais, os espécimes de $C$. heveae, Phyllocoptruta seringueirae Feres (Actinedida: Eriophyidae) e Tenuipalpus heveae Baker (Actinedida: Tenuipalpidae) foram contados diretamente nos folíolos, devido ao seu fácil reconhecimento. Uma parcela dos ácaros de cada espécie (50 ácaros por transecto/coleta) foi montada para a confirmação da identificação. As lâminas montadas foram mantidas em estufa a cerca de $55^{\circ} \mathrm{C}$ por até três dias. Posteriormente, foi feita a lutagem dos bordos da lamínula com resina alquídica $\left(\right.$ Verniz $\mathrm{Cristal}^{\circledR}$ ). O exame para a identificação dos espécimes foi feito sob microscópio óptico com contraste de fases.

Para verificar possível diferença no número de ácaros presentes entre os transectos, utilizou-se o teste G (Zar 1999). A similaridade da acarofauna em relação a distância da borda nas duas áreas foi determinada pela aplicação do índice de Bray-Curtis (Krebs 1999). A diversidade e a uniformidade da acarofauna foram analisadas através do índice de Shannon-Wiener (H') e de Pielou (e), respectivamente (Magurran 1988). A diversidade máxima teórica (H'max) foi determinada segundo Krebs (1999).
O material testemunho foi depositado na coleção de Acari (DZSJRP) - http://www.splink.cria.org.br, do Departamento de Zoologia e Botânica, Universidade Estadual Paulista (UNESP), São José do Rio Preto, São Paulo.

\section{Resultados}

O número de fitófagos foi menor no transecto próximo à vegetação nativa (transecto $\mathrm{I}$ ), tanto no fragmento de Cerradão $(\mathrm{G}=28.432,74$; d.f. $=4 ; \mathrm{P}<0,0001)$, quanto no de Mata Ripária $(\mathrm{G}=7.889,88$; d.f. $=4 ; \mathrm{P}<0,0001)$ (Figs. 1 e 2). $\mathrm{O}$ transecto $\mathrm{V}$ apresentou a maior riqueza de espécies no seringal vizinho ao fragmento de Cerradão (Tabela 1), enquanto que os transectos III e IV no seringal vizinho à Mata Ripária (Tabela 2). Entretanto, analisando as duas áreas juntas, os transectos I e IV apresentaram maior riqueza de espécies, enquanto o transecto III, a menor.

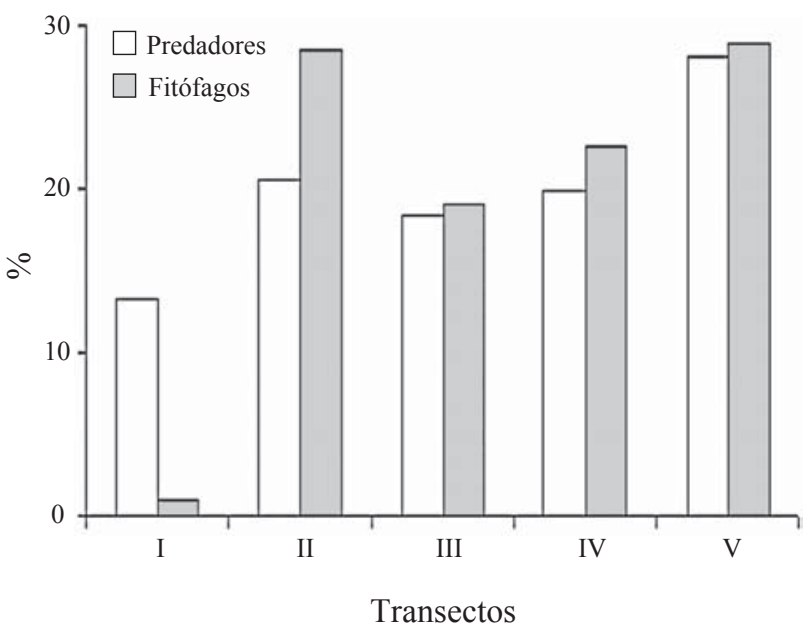

Fig. 1. Distribuição (\%) de ácaros predadores e fitófagos no seringal vizinho ao fragmento de Cerradão.

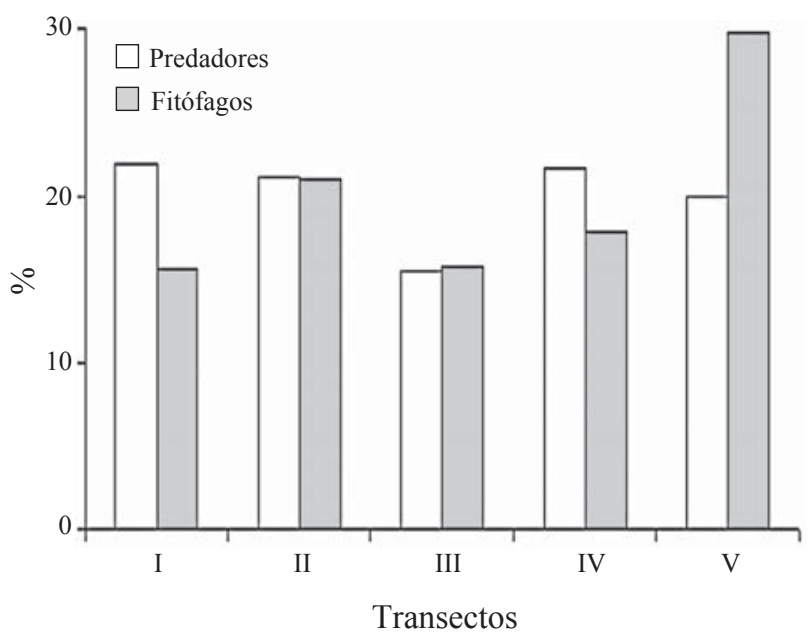

Fig. 2. Distribuição (\%) de ácaros predadores e fitófagos no seringal vizinho ao fragmento de Mata Ripária. 
Tabela 1. Abundância e distribuição das espécies de ácaros registradas nas plantas dos cinco transectos analisados no seringal vizinho ao fragmento de Cerradão.

\begin{tabular}{|c|c|c|c|c|c|c|c|}
\hline \multirow{2}{*}{ Espécies } & \multirow{2}{*}{ H.A. } & \multicolumn{5}{|c|}{ Transectos } & \multirow{2}{*}{ Total } \\
\hline & & I & II & III & IV & $\mathrm{V}$ & \\
\hline \multicolumn{8}{|l|}{ Acaridae } \\
\hline Caloglyphus sp. & M & 0 & 0 & 0 & 0 & 2 & 2 \\
\hline \multicolumn{8}{|l|}{ Bdellidae } \\
\hline $\begin{array}{l}\text { Hexabdella cinquaginta Hernandes, } \\
\text { Daud \& Feres }\end{array}$ & $\mathrm{P}$ & 0 & 0 & 0 & 0 & 1 & 1 \\
\hline \multicolumn{8}{|l|}{ Cunaxidae } \\
\hline Scirula sp. & $\mathrm{P}$ & 0 & 1 & 3 & 0 & 5 & 9 \\
\hline Neocunaxoides sp. & $\mathrm{P}$ & 0 & 0 & 1 & 0 & 0 & 1 \\
\hline \multicolumn{8}{|l|}{ Eriophyidae } \\
\hline Cf. Acarolox sp. & $\mathrm{F}$ & 0 & 0 & 0 & 0 & 1 & 1 \\
\hline Calacarus heveae Feres & $\mathrm{F}$ & 326 & 61 & 446 & 180 & 63 & 1.076 \\
\hline Phyllocoptruta seringueirae Feres & $\mathrm{F}$ & 128 & 16.630 & 11.768 & 14.168 & 17.718 & 60.412 \\
\hline Shevtchenkella petiolula Feres & $\mathrm{F}$ & 14 & 63 & 53 & 152 & 157 & 439 \\
\hline \multicolumn{8}{|l|}{ Oripodidae } \\
\hline Pirnodus sp. & M & 2 & 0 & 2 & 1 & 3 & 8 \\
\hline \multicolumn{8}{|l|}{ Phytoseiidae } \\
\hline $\begin{array}{l}\text { Amblyseius neochiapensis Lofego, } \\
\text { Moraes \& McMurtry }\end{array}$ & $\mathrm{PP}$ & 0 & 1 & 2 & 2 & 0 & 5 \\
\hline Euseius citrifolius Denmark \& Muma & PP & 3 & 6 & 4 & 1 & 6 & 20 \\
\hline Euseius concordis (Chant) & PP & 31 & 61 & 32 & 39 & 52 & 215 \\
\hline Iphiseiodes zuluagai Denmark \& Muma & PP & 7 & 12 & 5 & 9 & 17 & 50 \\
\hline Imaturos & & 13 & 15 & 29 & 20 & 26 & 103 \\
\hline \multicolumn{8}{|l|}{ Scheloribatidae } \\
\hline Scheloribates sp. & M & 0 & 0 & 1 & 0 & 0 & 1 \\
\hline \multicolumn{8}{|l|}{ Stigmaeidae } \\
\hline Agistemus sp. & $\mathrm{P}$ & 42 & 51 & 52 & 60 & 70 & 275 \\
\hline $\begin{array}{l}\text { Zetzellia quasagistemas Hernandes \& } \\
\text { Feres }\end{array}$ & $\mathrm{P}$ & 3 & 5 & 6 & 4 & 20 & 38 \\
\hline Imaturos & & 5 & 8 & 10 & 20 & 23 & 66 \\
\hline \multicolumn{8}{|l|}{ Tarsonemidae } \\
\hline Daidalotarsonemus tesselatus DeLeon & $?$ & 11 & 23 & 17 & 60 & 39 & 150 \\
\hline Tarsonemus confusus Ewing & M1 & 5 & 6 & 2 & 8 & 3 & 24 \\
\hline Tarsonemus sp.1 & $\mathrm{M}^{1}$ & 1 & 6 & 1 & 4 & 10 & 22 \\
\hline Tarsonemus sp. 2 & $\mathrm{M}^{1}$ & 3 & 4 & 1 & 4 & 6 & 18 \\
\hline Tarsonemus sp. 3 & $\mathrm{M}^{1}$ & 0 & 0 & 0 & 1 & 0 & 1 \\
\hline Xenotarsonemus sp. & $?$ & 0 & 0 & 0 & 0 & 1 & 1 \\
\hline Imaturos & & 5 & 12 & 21 & 11 & 15 & 64 \\
\hline \multicolumn{8}{|l|}{ Tenuipalpidae } \\
\hline Brevipalpus phoenicis (Geijskes) & $\mathrm{F}$ & 1 & 0 & 0 & 2 & 0 & 3 \\
\hline Tenuipalpus heveae Baker & $\mathrm{F}$ & 213 & 3.947 & 1.503 & 1.920 & 3.059 & 10.642 \\
\hline
\end{tabular}

Tetranychidae

10

0

0

$0 \quad 1$ 
Tabela 1. Continuação.

\begin{tabular}{|c|c|c|c|c|c|c|c|}
\hline \multirow{2}{*}{ Espécies } & \multirow{2}{*}{ H.A. } & \multicolumn{5}{|c|}{ Transectos } & \multirow{2}{*}{ Total } \\
\hline & & I & II & III & IV & $\mathrm{V}$ & \\
\hline Oligonychus gossypii (Zacher) & $\mathrm{F}$ & 30 & 57 & 25 & 56 & 25 & 193 \\
\hline Tetranychus mexicanus (McGregor) & $\mathrm{F}$ & 1 & 15 & 32 & 8 & 8 & 64 \\
\hline Imaturos & & 14 & 12 & 2 & 2 & 17 & 47 \\
\hline \multicolumn{8}{|l|}{ Tydeidae } \\
\hline Lorryia formosa Cooreman & $\mathrm{F}^{2}$ & 2 & 2 & 0 & 0 & 2 & 6 \\
\hline Lorryia sp. 2 & $\mathrm{~F}$ & 5 & 1 & 3 & 4 & 1 & 14 \\
\hline Metapronematus sp. & $?$ & 0 & 0 & 0 & 0 & 1 & 1 \\
\hline Neolorryia boycei (Baker) & $?$ & 0 & 1 & 1 & 0 & 1 & 3 \\
\hline Paralorryia sp. & $?$ & 0 & 1 & 0 & 0 & 0 & 1 \\
\hline Parapronematus sp. & $\mathrm{P}^{3}$ & 0 & 1 & 0 & 0 & 0 & 1 \\
\hline Pseudolorryia sp. & $?$ & 2 & 1 & 16 & 57 & 4 & 80 \\
\hline Imaturos & & 0 & 0 & 3 & 5 & 0 & 8 \\
\hline \multicolumn{8}{|l|}{ Winterschmidtiidae } \\
\hline Czenspinskia sp. & $\mathrm{M}^{4}$ & 2 & 0 & 0 & 2 & 0 & 4 \\
\hline Oulenzia sp. & $\mathrm{M}^{4}$ & 2 & 0 & 0 & 0 & 1 & 3 \\
\hline Abundância & & 872 & 21.003 & 14.041 & 16.800 & 21.357 & 74.073 \\
\hline Riqueza & & 23 & 23 & 22 & 22 & 27 & 36 \\
\hline
\end{tabular}

(H.A.) hábito alimentar: (F) fitófagos (Jeppson et al. 1975); (PP) preponderantemente predadores (Krantz 1978, McMurtry \& Croft 1997); (P) predadores (Krantz 1978, Flechtmann 1986), (M) micófagos; (?) existem dúvidas a respeito do hábito alimentar dessas espécies ou gêneros: (1) Lindquist 1986; (2) Smirnoff 1957, Gerson 1968, Flechtmann 1973, Flechtmann \& Arleu 1984; (3) McCoy et al. 1967; (4) Baker \& Wharton 1952, Krantz 1978, Woolley 1988.

Observou-se também baixa similaridade (10\%) do transecto I em relação aos demais transectos amostrados na área de cultivo vizinho ao fragmento de Cerradão (Fig. 3). No cultivo próximo ao fragmento de Mata Ripária a similaridade entre os transectos I e II foi de $85 \%$, ocorrendo a menor similaridade entre os transectos I e V (53\%) (Fig. 4).

O fitófago $P$. seringueirae teve a maior abundâncias registrada no transecto mais distante da mata nativa (transecto V) e a menor no mais próximo (transecto I), em ambos os seringais. Entretanto, T. heveae foi mais abundante no transecto localizado a $50 \mathrm{~m}$ da borda (transecto II). Com relação aos predadores, algumas espécies tiveram suas maiores abundâncias nos transectos localizados no interior do seringal.

A diversidade nas duas áreas foi menor que $50 \% \mathrm{em}$ relação à diversidade máxima teórica (Tabela 3). Contudo, no transecto I do cultivo próximo ao fragmento de Cerradão, esse índice foi de $57 \%$, com diversidade e uniformidade três vezes maiores em relação aos demais transectos. No transecto I da Mata Ripária a diversidade e uniformidade foram $70 \%$ maiores em relação ao transecto $\mathrm{V}$.

\section{Discussão}

As menores abundâncias de fitófagos foram registradas nas seringueiras do transecto no limite com as áreas nativas, fato este que pode estar relacionado ao efeito de borda. Ou seja, com a distância das seringueiras desses transectos em relação às áreas nativas vizinhas. Demite \& Feres (2005) também registraram a menor abundância de ácaros fitófagos em seringal no transecto próximo de fragmento de Mata Estacional Semidecidual, em São José do Rio Preto, SP.

A proximidade com áreas naturais, onde ocorre o deslocamento de ácaros predadores da vegetação de entorno para o seringal, contribui para manter as populações de fitófagos em níveis pequenos, além dos fatores abióticos que interferem no crescimento populacional das espécies-praga. Nas bordas, os níveis de luminosidade, temperatura e vento são maiores (Kapos 1989, Bierregaard et al. 1992, Rodrigues 1998). Queiroga (2000) verificou que próximo à borda de uma área de cerrado, a umidade do ar era mais baixa do que no seu interior.

A maior ocorrência de $T$. heveae nos transectos mais próximos das áreas nativas pode ser resultado do deslocamento competitivo provocado por $P$. seringueirae, fato este que deve ser estudado. $T$. heveae e $P$. seringueirae habitam o mesmo microhábitat, a face inferior dos folíolos. A maior abundância de algumas espécies de ácaros predadores nos transectos do interior do seringal provavelmente deve-se à maior oferta de presas, já que na borda do seringal o número de presas é menor, provavelmente devido aos fatores acima mencionados.

Os índices de similaridade entre os transectos foram afetados principalmente pela abundância das espécies fitófagas, principalmente no seringal vizinho ao Cerradão, onde se observou baixa similaridade do transecto da borda com os demais. 
Tabela 2. Abundância e distribuição das espécies de ácaros registradas nas plantas dos cinco transectos analisados no fragmento de Mata Ripária.

\begin{tabular}{|c|c|c|c|c|c|c|c|}
\hline \multirow{2}{*}{ Espécies } & \multirow{2}{*}{ H.A. } & \multicolumn{5}{|c|}{ Transectos } & \multirow{2}{*}{ Total } \\
\hline & & I & II & III & IV & $\mathrm{V}$ & \\
\hline \multicolumn{8}{|l|}{ Acaridae } \\
\hline Caloglyphus sp. & M & 46 & 49 & 2 & 67 & 0 & 164 \\
\hline \multicolumn{8}{|l|}{ Ascidae } \\
\hline Asca sp. & $\mathrm{P}$ & 0 & 1 & 0 & 1 & 0 & 1 \\
\hline \multicolumn{8}{|l|}{ Bdellidae } \\
\hline $\begin{array}{l}\text { Hexabdella cinquaginta Hernades, } \\
\text { Daud \& Feres }\end{array}$ & $P$ & 0 & 0 & 0 & 0 & 1 & 1 \\
\hline \multicolumn{8}{|l|}{ Cheyletidae } \\
\hline Cheletomimus duosetosus Muma & $\mathrm{P}$ & 41 & 61 & 16 & 46 & 9 & 173 \\
\hline Cheletomimus aff. darwinia & $P$ & 0 & 0 & 1 & 0 & 0 & 1 \\
\hline \multicolumn{8}{|l|}{ Cunaxidae } \\
\hline Scirula sp. & $\mathrm{P}$ & 60 & 39 & 54 & 84 & 72 & 309 \\
\hline \multicolumn{8}{|l|}{ Eriophyidae } \\
\hline Calacarus heveae Feres & $\mathrm{F}$ & 141 & 399 & 937 & 2.069 & 2.482 & 6.028 \\
\hline Phyllocoptruta seringueirae Feres & $\mathrm{F}$ & 13.549 & 18.962 & 17.022 & 18.182 & 33.101 & 100.816 \\
\hline Shevtchenkella petiolula Feres & $\mathrm{F}$ & 232 & 223 & 319 & 253 & 288 & 1.315 \\
\hline \multicolumn{8}{|l|}{ Oripodidae } \\
\hline Pirnodus sp. & M & 0 & 0 & 1 & 0 & 0 & 1 \\
\hline \multicolumn{8}{|l|}{ Phytoseiidae } \\
\hline Amblyseius acalyphus Denmark \& Muma & PP & 23 & 14 & 14 & 17 & 4 & 72 \\
\hline $\begin{array}{l}\text { Amblyseius neochiapensis Lofego, } \\
\text { Moraes \& McMurtry }\end{array}$ & PP & 9 & 3 & 1 & 11 & 0 & 24 \\
\hline Euseius citrifolius Denmark \& Muma & PP & 2 & 2 & 2 & 1 & 8 & 15 \\
\hline Euseius concordis (Chant) & PP & 5 & 13 & 21 & 13 & 7 & 59 \\
\hline Galendromus sp. & PP & 0 & 0 & 1 & 0 & 0 & 1 \\
\hline Iphiseiodes zuluagai Denmark \& Muma & PP & 0 & 0 & 2 & 0 & 1 & 3 \\
\hline Proprioseiopsis aff. jasmini & PP & 1 & 0 & 0 & 0 & 0 & 1 \\
\hline Typhlodromips aff. amilus & PP & 39 & 29 & 15 & 23 & 23 & 129 \\
\hline Imaturos & & 23 & 22 & 12 & 10 & 13 & 80 \\
\hline \multicolumn{8}{|l|}{ Scheloribatidae } \\
\hline Scheloribates sp. & M & 0 & 0 & 1 & 0 & 0 & 1 \\
\hline \multicolumn{8}{|l|}{ Stigmaeidae } \\
\hline Agistemus sp. & $P$ & 25 & 44 & 24 & 18 & 31 & 142 \\
\hline $\begin{array}{l}\text { Zetzellia quasagistemas Hernandes } \\
\text { \& Feres }\end{array}$ & $\mathrm{P}$ & 6 & 10 & 15 & 16 & 45 & 92 \\
\hline Imaturos & & 19 & 9 & 4 & 8 & 16 & 56 \\
\hline \multicolumn{8}{|l|}{ Tarsonemidae } \\
\hline Daidalotarsonemus tesselatus DeLeon & $?$ & 28 & 15 & 30 & 35 & 33 & 141 \\
\hline Deleonia sp. & $?$ & 0 & 0 & 1 & 0 & 0 & 1 \\
\hline Tarsonemus sp.2 & $M^{1}$ & 0 & 0 & 0 & 2 & 9 & 11 \\
\hline Xenotarsonemus sp. & $?$ & 1 & 0 & 0 & 0 & 0 & 1 \\
\hline Imaturos & & 12 & 9 & 8 & 10 & 4 & 43 \\
\hline
\end{tabular}


Tabela 2. Continuação.

\begin{tabular}{|c|c|c|c|c|c|c|c|}
\hline \multirow{2}{*}{ Espécies } & \multirow{2}{*}{ H.A. } & \multicolumn{5}{|c|}{ Transectos } & \multirow{2}{*}{ Total } \\
\hline & & I & II & III & IV & $\mathrm{V}$ & \\
\hline \multicolumn{8}{|l|}{ Tenuipalpidae } \\
\hline Tenuipalpus heveae Baker & $\mathrm{F}$ & 5.315 & 6.162 & 1.163 & 1.338 & 770 & 14.748 \\
\hline \multicolumn{8}{|l|}{ Tetranychidae } \\
\hline Aponychus cf. shultzi & $\mathrm{F}$ & 1 & 0 & 0 & 0 & 0 & 1 \\
\hline Oligonychus gossypii (Zacher) & $\mathrm{F}$ & 51 & 68 & 84 & 138 & 53 & 394 \\
\hline Tetranychus mexicanus (McGregor) & $\mathrm{F}$ & 25 & 67 & 6 & 65 & 51 & 214 \\
\hline Imaturos & & 5 & 24 & 35 & 36 & 31 & 131 \\
\hline \multicolumn{8}{|l|}{ Tydeidae } \\
\hline Lorryia formosa Cooreman & $\mathrm{F}^{2}$ & 4 & 2 & 3 & 1 & 4 & 14 \\
\hline Lorryia sp.1 & $\mathrm{F}$ & 0 & 0 & 0 & 2 & 0 & 2 \\
\hline Lorryia sp. 2 & $\mathrm{~F}$ & 2 & 8 & 3 & 2 & 3 & 18 \\
\hline Metapronematus sp. & $?$ & 5 & 2 & 1 & 2 & 0 & 10 \\
\hline Neolorryia boycei (Baker) & $?$ & 0 & 1 & 3 & 1 & 1 & 6 \\
\hline Parapronematus sp. & $\mathrm{P}^{3}$ & 2 & 0 & 0 & 0 & 0 & 2 \\
\hline Pretydeus sp. & $?$ & 0 & 0 & 0 & 1 & 0 & 1 \\
\hline Pronematus sp. & $\mathrm{P}^{4}$ & 2 & 1 & 0 & 3 & 3 & 9 \\
\hline Pseudolorryia sp. & $?$ & 9 & 6 & 4 & 7 & 11 & 37 \\
\hline Imaturos & & 0 & 3 & 2 & 11 & 7 & 23 \\
\hline \multicolumn{8}{|l|}{ Winterschmidtiidae } \\
\hline Czenspinskia sp. & $\mathrm{M}^{5}$ & 0 & 0 & 0 & 0 & 1 & 1 \\
\hline Oulenzia sp. & $\mathrm{M}^{5}$ & 0 & 9 & 4 & 1 & 1 & 15 \\
\hline Abundância & & 19.683 & 26.257 & 19.811 & 22.474 & 37.083 & 125.308 \\
\hline Riqueza & & 26 & 25 & 28 & 28 & 24 & 40 \\
\hline
\end{tabular}

(H.A.) hábito alimentar: (F) fitófagos (Jeppson et al. 1975); (PP) preponderantemente predadores (Krantz 1978, McMurtry \& Croft 1997); (P) predadores (Krantz 1978, Flechtmann 1986), (M) micófagos; (?) existem dúvidas a respeito do hábito alimentar dessas espécies ou gêneros: (1) Lindquist 1986; (2) Smirnoff 1957, Gerson 1968, Flechtmann 1973, Flechtmann \& Arleu 1984; (3) McCoy et al. 1967; (4) Baker 1965, Baker \& Wharton 1952, Bayan 1986, Calvert \& Huffaker 1974, Knop \& Hoy 1983, Laing \& Knop 1983, Perrin \& McMurtry 1996; (5) Baker \& Wharton 1952, Krantz 1978, Woolley 1988.

A diversidade e a uniformidade foram baixas nas seringueiras amostradas nas duas áreas devido à grande dominância de $P$. seringueirae e $T$. heveae, espécies mais abundantes nas seringueiras (Demite \& Feres 2007). O mesmo padrão foi verificado em trabalhos realizados no estado de São Paulo, onde a ocorrência de grande população de $C$. heveae provocou baixos níveis de uniformidade e conseqüentemente, de diversidade (Feres et al. 2002, Bellini et al. 2005, Demite \& Feres 2005). A diversidade costuma ser maior em ecossistemas naturais que apresentam grande estabilidade, enquanto em ecossistemas com interferência antrópica, a diversidade e a uniformidade são baixas (Odum 1988). Ambientes mais homogêneos dificultam o estabelecimento de inimigos naturais, maiores responsáveis pelo controle biológico (Risch 1981, Andow \& Risch 1985, Andow 1991). Segundo Altieri et al. (2003), agroecossistemas apresentam menor heterogeneidade ambiental que ecossistemas naturais, tendo as espécies fitófagas maior possibilidade de alcançar altos níveis populacionais, gerando assim pequenos índices de uniformidade. Esses mesmos autores sugerem o aumento da heterogeneidade dentro e próximo de agroecossistemas, com a presença de vegetação adjacente, plantas "invasoras", etc. De acordo com Huston (1995), quanto maior a heterogeneidade ambiental, maior será a diversidade da fauna.

Os índices de uniformidade e diversidade nos transectos foram maiores nas seringueiras mais próximas às áreas nativas (transecto I) do que nos demais transectos, principalmente comparando com aqueles mais distantes da vegetação. No transecto I do seringal próximo ao fragmento de Cerradão, a diversidade atingiu mais da metade da diversidade máxima esperada, enquanto que no transecto $\mathrm{V}$, somente $17 \%$. Esta maior diversidade no transecto mais próximo da vegetação nativa deve-se à menor abundância dos ácaros fitófagos. Faixas de vegetação marginal talvez representem a forma mais promissora para a introdução de biodiversidade na paisagem agrícola, com benefícios para o controle de pragas (Altieri et al. 2003). 
O conhecimento básico em relação às plantas que podem atuar como reservatório e a distribuição de ácaros dentro da cultura, pode fornecer dados para elaboração de programas de manejo ecológico a serem implantados com sucesso no futuro.

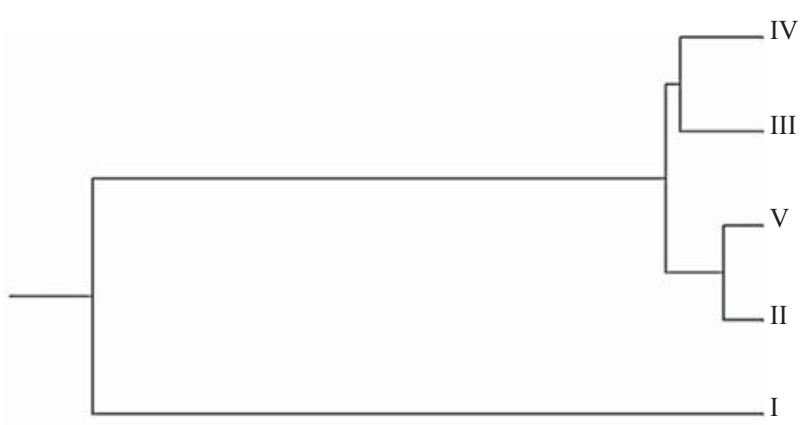

$\%$ Similaridade

50

100

Fig. 3. Similaridade (Bray-Curtis) acarofaunística entre os transectos analisados no cultivo vizinho ao fragmento de Cerradão.

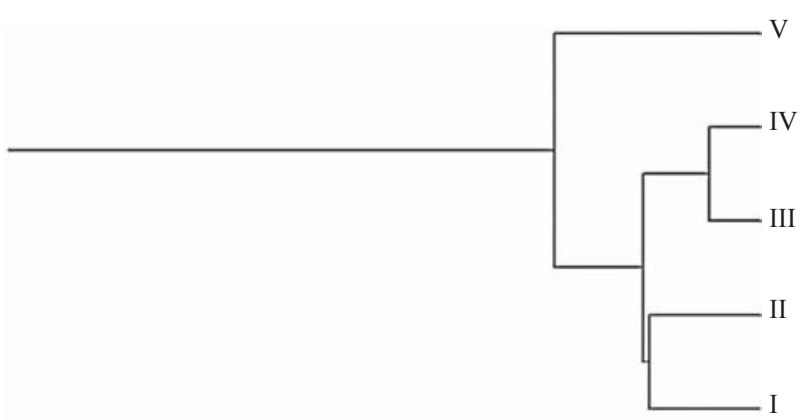

$\%$ Similaridade 50 100

Fig. 4. Similaridade (Bray-Curtis) acarofaunística entre os transectos analisados no cultivo vizinho ao fragmento de Mata Ripária.
Demite et al. (submetido), em levantamento da acarofauna associada às plantas dos fragmentos vizinhos aos seringais estudados, registraram dez espécies de ácaros predadores que ocorreram também nas seringueiras amostradas. Demite \& Feres (2005), relatam a ocorrência de cinco espécies de ácaros predadores, coletados em seringueiras e em plantas nativas vizinhas às seringueiras amostradas. Esses dados sugerem que as áreas nativas podem servir de reservatórios de ácaros predadores, que podem se deslocar para as seringueiras em função da oferta de alimento.

Jung \& Croft (2001) verificaram a dispersão aérea de algumas espécies de Phytoseiidae que colonizaram vinhedos a partir da vegetação natural de entorno, sendo que a colonização estava diretamente associada com a abundância de fitoseídeos e a proximidade da vegetação natural (Tixier et al. 1998 e 2000).

Áreas nativas são benéficas devido à oferta de pólen, néctar, abrigo, locais de reprodução, presas e hospedeiros alternativos para inimigos naturais durante períodos em que esses recursos são escassos nas culturas onde eles supostamente devem controlar as pragas (Chiverton \& Sotherton 1991). Além disso, áreas nativas também podem fornecer locais de refúgio para os predadores durante períodos de aplicação de produtos químicos em cultivos vizinhos (Smith \& Papacek 1991).

Uma rede de áreas nativas como cercas vivas, faixas preservadas de mata nativa, refúgios constituídos de vegetação específica que atraia presas alternativas e áreas de mata ciliar, podem ampliar o movimento dos inimigos naturais entre os elementos da paisagem, melhorando a acessibilidade a esses elementos e provendo muitos recursos para a entrada de espécies benéficas nos agroecossistemas (Riffel \& Gutzwiller 1996). Segundo Baxter (1979) apud Altieri et al. (2003) os maiores níveis de controle biológico ocorrem nas bordas das culturas vizinhas à vegetação nativa.

Os dados apresentados reforçam a importância da existência de vegetação natural vizinha à áreas de cultivo de seringueira, sendo este um fator importante a ser considerado na elaboração de programas de manejo ecológico de pragas. Além disso, sugerem que em uma faixa de borda de aproximadamente $50 \mathrm{~m}$ no seringal, próximo de áreas nativas, deve ser evitada a aplicação de agrotóxicos, protegendo-se a fauna de ácaros predadores, reduzindo a contaminação ambiental e humana.

Tabela 3. Diversidade (H'), diversidade máxima teórica (H'max) e uniformidade (e) da fauna de ácaros nos transectos dos dois seringais estudados, vizinhos de fragmentos de cerrado.

\begin{tabular}{llllllll}
\hline Área & Índice & I & II & III & IV & V & Geral \\
\hline \multirow{3}{*}{ Cerradão } & H' & 0,77 & 0,27 & 0,26 & 0,26 & 0,25 & 0,28 \\
& H'max & 1,36 & 1,36 & 1,34 & 1,34 & 1,43 & 1,54 \\
& $\mathrm{e}$ & 0,57 & 0,20 & 0,19 & 0,19 & 0,17 & 0,18 \\
\hline \multirow{3}{*}{ Mata Ripária } & H' & 0,36 & 0,34 & 0,26 & 0,33 & 0,20 & 0,32 \\
& H'max & 1,41 & 1,39 & 1,56 & 1,45 & 1,38 & 1,60 \\
& $\mathrm{e}$ & 0,25 & 0,24 & 0,18 & 0,23 & 0,15 & 0,20 \\
\hline \multirow{3}{*}{ Duas áreas } & H' & 0,40 & 0,31 & 0,27 & 0,31 & 0,24 & 0,31 \\
& H'max & 1,54 & 1,49 & 1,52 & 1,54 & 1,52 & 1,68 \\
& $\mathrm{e}$ & 0,26 & 0,21 & 0,18 & 0,20 & 0,16 & 0,18 \\
\hline
\end{tabular}




\section{Agradecimentos}

Ao Prof. Dr. Antonio C. Lofego (Depto. Zoologia e Botânica, UNESP, São José do Rio Preto), pela identificação dos tarsonemídeos e auxílio na identificação dos fitoseídeos. Ao Dr. Aníbal R. de Oliveira (Depto. de Ciências Agrárias e Ambientais, Universidade Estadual de Santa Cruz, Ilhéus, BA), pela identificação dos oribatídeos. Ao Prof. Dr. Francisco Langeani Neto e ao Dr. Gustavo Quevedo Romero (Depto. Zoologia e Botânica, UNESP, São José do Rio Preto), pelas críticas e sugestões ao manuscrito. À Plantações E. Michelin Ltda., Itiquira, MT, pela bolsa concedida ao autor sênior e pelo financiamento do projeto; e aos engenheiros agrônomos dessa empresa, Cássio J. H. Scomparim e Fernando S. Fonseca, pelas informações fornecidas a respeito dos locais de coleta e pelo apoio durante o andamento do projeto.

\section{Referências}

Altieri, M.A. 1994. Biodiversity and pest management in agroecosystems. New York, Food Products Press, 185p.

Altieri, M.A. 1999. The ecological role of biodiversity in agroecosystems. Agric. Ecosyst. Environ. 74: 19-31.

Altieri, M.A., N.E. Silva \& C.I. Nicholls. 2003. O papel da biodiversidade no manejo de pragas. Ribeirão Preto, Editora Holos Ltda, 226p.

Andow, D.A. 1991. Vegetation diversity and arthropod population response. Annu. Rev. Entomol. 36: 561-586.

Andow, D.A. \& S.J. Risch. 1985. Predation in diversified agroecosystems: Relations between a coccinellid predator and its food. J. Appl. Ecol. 22: 357-372.

Baker, E.W. 1965. A review of the genera of the family Tydeidae (Acarina). Adv. Acarol. 2: 95-133.

Baker, E.W. \& A.E. Wharton. 1952. An introduction to acarology. New York, MacMillan Co., 465p.

Bayan, A. 1986. Tydeidae mites associated with apples in Lebanon (Acari: Actinedida: Tydeidae). Acarologia 27: 311-316.

Bellini, M.R., G.J. de Moraes, R.J.F. Feres. 2005. Ácaros (Acari) de dois sistemas comuns de cultivo de seringueira no noroeste do estado de São Paulo. Neotrop. Entomol. 34: 475-484

Bierregaard, R.O. Jr., T.E. Lovejoy, V. Kapos, A.A. dos Santos \& R.W. Hutchings. 1992. The biological dynamics of tropical rainforest fragments. Bioscience 42: 859-866.

Calvert, D.J. \& C.B. Huffaker. 1974. Predator (Metaseiulus occidentalis) - prey (Pronematus spp.) interactions under sulfur and cattail pollen applications in a noncommercial vineyard. Entomophaga 19: 361-369.

Chiverton, P.A. \& N.W. Sotherton. 1991. The effects on beneficial arthropods of the exclusion of herbicides from cereal crops edges. J. App. Ecol. 28: 1027-1039.

Demite, P.R. \& R.J.F. Feres. 2005. Influência de vegetação vizinha na distribuição de ácaros (Acari) em seringal no município de
São José do Rio Preto, São Paulo, Brasil. Neotrop. Entomol. 34: 829-836.

Demite, P.R. \& R.J.F. Feres. 2007. Ocorrência e flutuação populacional de ácaros associados a seringais vizinhos de fragmentos de cerrado. Neotrop. Entomol. 36: 117-127.

Demite, P.R., R.J.F. Feres, A.C. Lofego \& A.R. Oliveira. Ácaros de plantas do cerrado mato-grossense. Biota Neotropica (submetido para publicação).

Feres, R.J.F., D. de C. Rossa-Feres, R.D. Daud \& R.S. Santos. 2002. Diversidade de ácaros (Acari, Arachnida) em seringueiras (Hevea brasiliensis Muell. Arg., Euphorbiaceae) na região noroeste do estado de São Paulo, Brasil. Rev. Bras. Zool. 19: 137-144.

Flechtmann, C.H.W. 1973. Lorryia formosa Cooreman, 1958 Um ácaro do citros pouco conhecido no Brasil. Cienc. Cult. 25:1179-1181.

Flechtmann, C.H.W. 1975. Elementos de acarologia. São Paulo, Livraria Nobel S.A., 344p.

Flechtmann, C.H.W. 1986. Ácaros em produtos armazenados e na poeira domiciliar. Piracicaba, Livraria Nobel S.A., 344p.

Flechtmann, C.H.W. \& R.J. Arleu. 1984. Oligonychus coffeae (Nietner, 1861), um ácaro tetraniquídeo para a seringueira (Hevea brasiliensis) novo para o Brasil e observações sobre outros ácaros desta planta. Ecossistema 9: 123-125.

Gerson, U. 1968. Five tydeid mites from Israel (Acari: Prostigmata). Isr. J. Zool. 17: 191-198.

Huston, M.A. 1995. Biological diversity: The coexistence of species on changing landscapes. Cambridge, Cambridge University Press, 681p.

Jeppson, L.R., H.H. Keifer \& E.W. Baker. 1975. Mites injurious to economic plants. Los Angeles, Univ. Calif. Press, 614pp $+74 \mathrm{pl}$.

Jung, C. \& B.A. Croft. 2001. Aerial dispersal of phytoseiid mites (Acari: Phytoseiidae): estimating falling speed and dispersal distance of adult females. Oikos 94: 182-190.

Kapos, V. 1989. Effects of isolation on the water status of forest patches in the Brazilian Amazon. J. Trop. Ecol. 5: 173-185.

Knop, N.F. \& M.A. Hoy. 1983. Factors limiting the utility of Homeopronematus anconai (Acari: Tydeidae) in integrated pest management in San Joaquin Valley vineyards. J. Econ. Entomol. 76: 1181-1186.

Krantz, G.W. 1978. A manual of acarology. Corvallis, Oregon State University Book Stores, 509p.

Krebs, C.J. 1999. Ecological methodology. Menlo Park, Ed. Adson Wesley Longman Inc., 620p.

Laing, J.E. \& N.F. Knop. 1983. Potencial use of predaceous mites other than Phytoseiidae for biological control of orchard pests, p.28-35. In M.A. Hoy, G.L. Cunningham \& L. Knustson (eds.), Biological control of pests by mites. University of California, Special Publication, Berkeley, no. 3304. 
Landis, D.A., S.D. Wratten \& G.M. Gurr. 2000. Habitat management to conserve natural enemies of arthropod pests in agriculture. Annu. Rev. Entomol. 45: 175-201.

Lindquist, E.E. 1986. The world genera of Tarsonemidae (Acari: Heterostigmata): A morphological, phylogenetic and systematic revision, with reclassification of family group taxa in Heterostigmata. Mem. Entomol. Soc. Can. 136: 1-517.

Magurran, A.E. 1998. Ecological diversity and its measurement. Cambridge, University Press, 179p.

McCoy, C.W., A.G. Selhime \& R.F. Kanavel. 1967. The feeding behavior and biology of Parapronematus acaciae (Acarina: Tydeidae). Fla. Entomol. 52: 13-19.

McMurtry, J.A. \& B.A. Croft. 1997. Life-styles of phytoseiid mites and their roles in biological control. Annu. Rev. Entomol. 42: 291-321.

Odum, E.P. 1988. Ecologia. Rio de Janeiro, Editora Guanabara, $432 \mathrm{p}$.

Perrin, T.M. \& J.A. McMurtry. 1996. Other predatory arthropods, p.471-479. In E.E. Lindquist, M.W. Sabelis \& J. Bruin (eds.), World crop pests. Eriophyoid mites - their biology, natural enemies and control. Amsterdam, Elsevier Society, 643p.

Queiroga, J. L. de. 2000. Efeito de borda em fragmentos de cerrado em área de agricultura do Maranhão, Brasil. Dissertação de Mestrado, UEL, Londrina, 32p.

Ribeiro, J.F. \& B.M.T. Walter. 1998. Fitofisionomias do bioma cerrado, p.89-166. In S.M. Sano \& S.P. de Almeida (ed.), cerrado: Ambiente e flora. Planaltina, Embrapa-CPAC, 556p.

Riffel, S.K. \& K.J. Gutzwiller. 1996. Plant-species richness in corridor intersections: Is intersection shape influential? Landsc. Ecol. 11: 157-168.
Risch, S.J. 1981. Insect herbivore abundance in tropical monocultures and polycultures: An experimental test of two hypotheses. Ecology 62: 1325-1340.

Rodrigues, E. 1998. Edge effects on the generation of forest fragments in South Brazil, PR. Tese de Ph.D. Havard University, Cambridge, Massachusetts, 172p.

Smirnoff, W.A. 1957. An undescribed species of Lorryia (Acari: Tydeidae) causing injury to citrus trees in Marroco. J. Econ. Entomol. 50: 361-362.

Smith, D. \& D.F. Papacek. 1991. Studies of the predatory mite Amblyseius victoriensis (Acarina: Phytoseiidae) in citrus orchards in south-east Queensland: Control of Tegolophus australis and Phyllocoptruta oleivora (Acarina: Eriophyidae), effects of pesticides, alternative host plants and augmentative release. Exp. Appl. Acarol. 12: 195-217.

Tixier, M.-S., S. Kreiter \& P. Auger. 2000. Colonization of vineyards by phytoseiid mites: Their dispersal patterns in the pot and their fate. Exp. Appl. Acarol. 24: 191-211.

Tixier, M.-S., S. Kreiter, P. Auger \& M. Weber. 1998. Colonization of Langhedoc vineyards by phytoseiid mites (Acari: Phytoseiidae): Influence of wind and crop environment. Exp. Appl. Acarol. 22: 523-542.

Woolley, T.A. 1988. Acarology: Mites and human welfare. Fort Collins, Colorado, Library of Congress Cataloging in Publication, 484p.

Zar, J.H. 1999. Biostatistical analysis. New Jersey, Prentice-Hall, $663 p+212 p$

Received 14/XII/06. Accepted 06/XI/07. 